\title{
Dancing shapes: A comparison of luminance-induced distortions
}

\author{
Rob van Lier, Árpád Csathó \\ Nijmegen Institute for Cognition and Information (NICl), Radboud University Nijmegen, PO Box 9104, \\ NL-6500 HE Nijmegen, The Netherlands; - also Institute of Behavioral Sciences, University of Pécs, \\ Hungary; e-mail: r.vanlier@nici.kun.nl; websites: http://www.nici.ru.nl/People/LiervanRJ/index.html, \\ http://www.nici.ru.nl/ Robvl \\ Received 14 July 2004, in revised form 12 May 2005; published online 10 May 2006
}

\begin{abstract}
We present an illusory display in which a grid of outlined squares is positioned in front of a moving luminance gradient. Observers perceive a strong, illusory, 'wavelike' motion of the superimposed squares. We compared luminance effects on dynamic and static aspects of this illusion. The dynamic aspect was investigated by means of a temporal gradient, which induced an illusory pulsing of the outlined squares. The static aspect was investigated in two different ways. In one experiment, the outlined squares were positioned on a spatial gradient, which caused the squares to look like trapezoid shapes. In another experiment, the squares were positioned on different luminance fields, which affected their apparent size. In all experiments, luminance settings were the same, and observers were asked to indicate the direction and strength of the induced distortions. The overall results show large agreements between the dynamic distortion and the first-mentioned static distortion, whereas different tendencies emerged for the second static distortion. In a second series of experiments, we examined these distortions for various ranges of the luminance gradient and for border gradients as well. On the basis of these data, we explored how the directions of the perceived distortions of the single-gradient displays examined in this paper could be related to each other.
\end{abstract}

\section{Introduction}

It is well known that brightness differences can induce spatial distortions (eg Gregory and Heard 1983; Woodhouse and Taylor 1987; Kitaoka 1998; Roncato 2000). Brightness differences might modulate motion salience and induce illusory motion as well (Anstis and Rogers 1975, 1986; Gregory and Heard 1983; Mather 1984; Anstis et al 2000; Anstis 2001; Cavanagh and Anstis 2002). Here we introduce a vivid illusory display in which a moving gradient induces spatial distortions and illusory motion (see figure 1a/animation 1a). The display consists of grey squares with either dark or light contours, all superimposed on a cyclic luminance gradient. At the instant the gradient is set to move from left to right, each single square seems to 'come alive'. Observing this display, one may experience a forward-backward pulsing and small left-right movements of the squares. In addition, each square seems to wiggle about its vertical axis. The combination of these aspects gives the display with the moving gradient an overall lively appearance. In figure $1 \mathrm{~b} /$ animation $1 \mathrm{~b}$, an additional example is given in which the configuration of squares has been rotated by $90^{\circ}$ with respect to the background. When there is no motion of the background gradient (ie in the static displays), the squares appear to be slightly deformed and they seem to have different sizes, depending on their position with respect to the background gradient.

The illusory motion in the above displays seems to be related to the phenomenon described by Gregory and Heard (1983). Their stimuli consisted of two grey rectangles, one positioned above the other, each with two edges with a different luminance. In one of the rectangles, the left edge was relatively light and the right edge was relatively dark - when compared to the rectangle's inside. In the other rectangle this was the other way round. When the background luminance increased and decreased, 


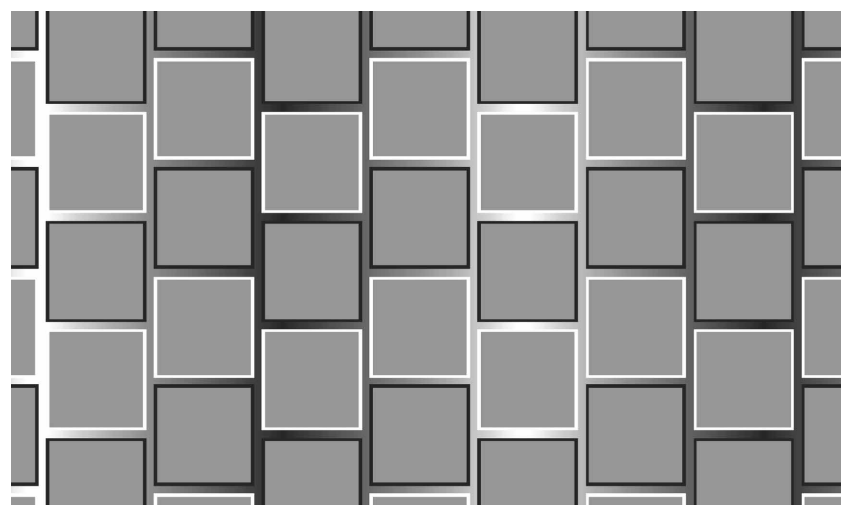

(a)

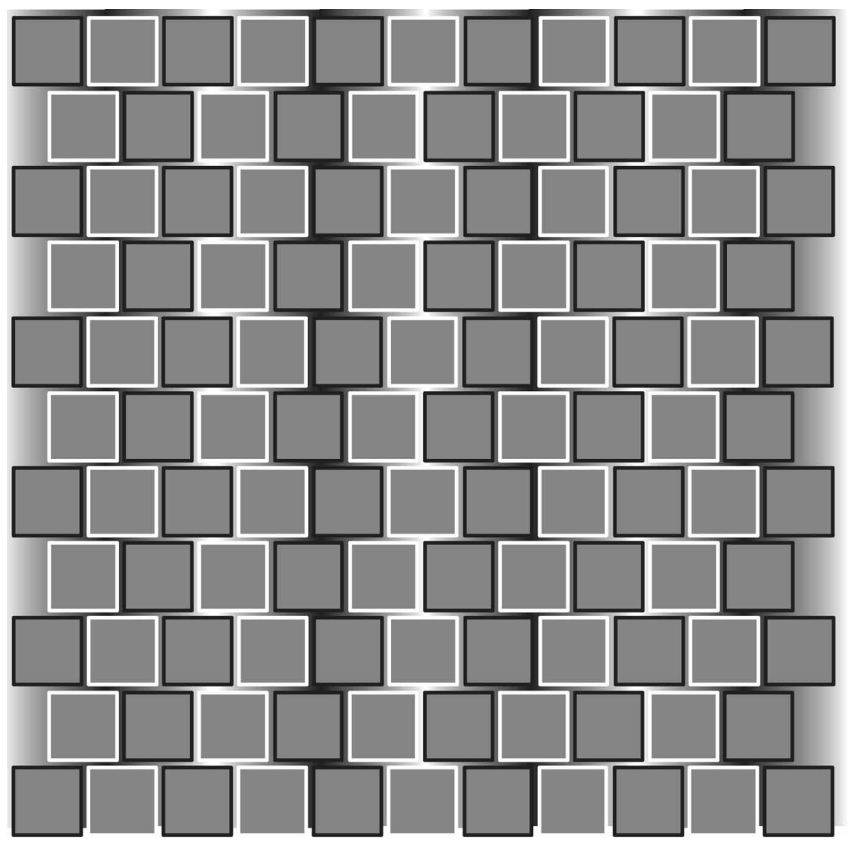

(b)

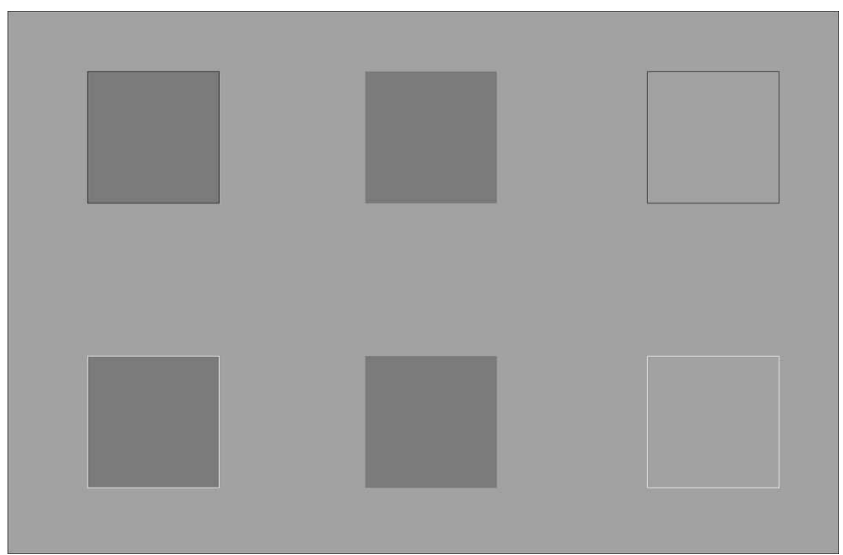


Figure 1 (animations). Examples of dancing shapes. (Note: Animations can be viewed on the Perception website at http://www.perceptionweb.com/misc/p5319a/ and should be run via Quicktime Player with the Movie > Loop option.) In animations (a) and (b) only the luminance gradient in the background is moving, yet there is an overall lively appearance of moving squares. In animation (c) the homogeneous background evolves from dark to light and vice versa; motion can clearly be observed in the squares with black or white borders (left column), and much less so, or not at all, in the non-bordered surfaces (middle column) or single outlines (right column). Note that the blackbordered surfaces seem to expand when the background changes from light to dark, whereas the white-bordered surfaces seem to expand when the background changes from dark to light (see text for further explanations). Further notice that the white-bordered surfaces actually seem smaller on a white background, although they give a clear impression of growing (or forward movement) when the background increases from dark to light (and, again, the reverse seems to hold for the black-bordered surfaces).

a left - right illusory motion of the rectangles was observed. Gregory and Heard reported that in the perceived motion the light edges were 'leading' when the background luminance increased and the dark edges were leading when the background luminance decreased. In addition, Gregory and Heard tested the apparent displacements in static displays. Here, they found that a rectangle with a light edge on one side and a dark edge on the other side appeared displaced toward the side with the light edge when the background luminance decreased and vice versa. The displacements in the dynamic displays and the static display apparently point in opposite directions. Gregory and Heard (1983) therefore concluded that the processing of motion and the spatial shifts they measured are dissociated and therefore rely on different perceptual mechanisms.

In the dynamic version of figure 1a, one may further observe that in each column the pulsing movements of adjacent squares are in antiphase with respect to each other: the squares with the light contours pulse forward when the lighter region of the gradient passes and the squares with the dark contours pulse forward when the darker region of the gradient passes. In the dynamic version of figure $1 \mathrm{~b}$, where the gradient runs parallel to the 'zig-zag' columns of squares with the same border, these columns seem much more to make the pulsing movements as a group.

Obviously, when the spatial frequency of the moving gradient would be much lower, the phenomenon would approach a purely temporal fluctuation of the background luminance, comparable to Gregory and Heard's (1983) experiment. Their observation that the light border was leading when the background luminance increases apparently agrees with the present extension (or growing, or forward pulsing) of the light-bordered squares at the instant the background gets lighter. Because of the four light edges (left, right, top, and bottom) - compared to only one light edge in Gregory and Heard's stimuli (on the left or right side) - a motion in all these directions occurs (which then is seen as a growing or forward motion). The animation of figure 1c shows the effect of the homogeneous temporal luminance gradient on the perceived movement (in this animation, also a surface without border and a border without inner surface have been added; one may observe that in the latter two cases there is hardly any pulsing visible-just a flicker near the onset of isoluminance). One may further notice that the white-bordered surfaces actually seem smaller on a white background although they give a clear impression of growing (or forward movement) when the background changes from dark to light (and, again, the reverse seems to hold for the black-bordered surfaces). This opposite direction seems to resemble the opposite displacements in Gregory and Heard's (1983) experiments.

The deformations that can be seen in the static displays of figures $1 \mathrm{a}$ and $1 \mathrm{~b}$ are quite similar to those investigated by Roncato (2000) in an extensive study. Roncato studied distortions of grey edges towards relatively dark or light adjacent regions. For these kinds of displays, Roncato demonstrated that the spatial distortion of such edges depends on the luminance profile of the regions adjacent to that edge. 
Amongst others, Roncato noted that, when a grey contour on a light background encountered an adjacent dark region, that grey contour shifted to the dark region; and, when the same contour on a dark background encountered an adjacent light region, it shifted to the lighter region. In addition, when one side of the contour was of a homogeneous grey and the other side had a variable luminance profile, the grey contour always deviated toward the region of luminance change. Roncato remarked that the above distortions were quite different from the aforementioned static displacements measured by Gregory and Heard (1983).

In contrast with the apparent size, the shape distortions due to the spatial gradient seem to point in the same direction as the dynamic growth and shrinkage: enlargement within a grey object at positions where its white contours are on a lighter background or at positions where black contours are on a darker background. In figure 2, the perceived difference in length between the left and right side of a square can be compared with the apparent size differences. In the upper row of squares, the border is relatively light; whereas in the lower row of squares, the border is relatively dark. In the upper row, the light-bordered square seems to be smaller on the light background than on the dark background. However, when the same square is positioned on a light-dark luminance gradient (see middle square) it appears larger on the light side and smaller on the dark side. For the lower row of squares, these effects are in the opposite direction.

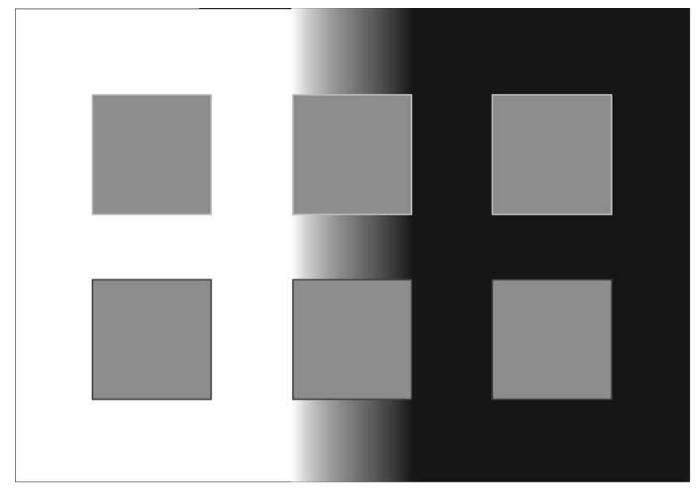

Figure 2. In the upper row, the central white-bordered square appears longer on the left side than on the right side. Note that the longer left side is on the white side of the gradient, whereas the shorter right side is on the black side of the gradient. Note further, however, that the left white-bordered square on the homogeneous white background appears smaller than the right white-bordered square on the homogeneous black background. In the lower row of squares, an opposite tendency is observed for the black-bordered square. Similar (opposite) distortion directions can also be observed in the dynamic case displayed in animation 1c.

Whereas Roncato (2000) noted the difference between the perceived displacements in his static displays and the displacements such as the ones reported in the Gregory and Heard (1983) study, he did not mention potential similarities between his own static displays and the motion direction in Gregory and Heard's (1983) dynamic displays. Conversely, Gregory and Heard's dissociation between the displacements in their dynamic and static displays could not be generalised to all static luminance-induced distortions. The present experiments have been set up to test and compare the combined influence of the luminances of border and surface on the direction and perceived strength of the size distortion in both the temporal and the spatial domain, when using the same luminance values. 


\section{Experiment 1}

The first experiment comprised three different sessions, referred to as experiment 1a, 1b, and 1c. Experiment 1a deals with the dynamic distortion (temporal domain), whereas experiments $1 \mathrm{~b}$ and $1 \mathrm{c}$ deal with the two static distortions discussed above (spatial domain). All luminance values were the same in each of the three experiments. Experiment la is based on the observation that the squares seem to extend and contract (or move backward and forward) when the luminance gradient passes. Experiment $1 \mathrm{~b}$ focuses on the shape deformation due to the gradient, whereas experiment 1c focuses on the subjective size differences of the outlined squares. The primary goal here is to compare the strengths of each of these illusory deformations produced by the same kinds of stimuli for these effects and the same luminance values in highly comparable tasks. The general method was similar in the three experiments. In each experiment, the stimuli consisted of an outlined square surrounded by a second surface. Either the inside of the square or the outside of the square could have a variable luminance. In experiments $1 \mathrm{a}$ and $1 \mathrm{~b}$ this was a temporal and spatial gradient, respectively, whereas in experiment $1 \mathrm{c}$ the luminance variation consisted of different luminance settings. In the following, the surface with a variable luminance is referred to as VLR (variable luminance region) and the surface with a constant luminance is referred to as CLR (constant luminance region).

\subsection{Participants}

Twelve subjects participated in all three sessions (experiments 1a-1c). All subjects had correct or corrected-to-normal vision. Half of the subjects first participated in the dynamic version (1a) and then in the two static versions (1b and 1c). The other half proceeded in a reversed order.

\subsection{Apparatus}

The experiment was run on a Pentium 4 configuration with a Radius Monitor, and was programmed in the 'Open-GL' environment. The luminances were measured with a Pritchard photometer.

\subsection{Stimuli and procedure}

2.3.1 Experiment 1a. Subjects viewed a stimulus consisting of a homogeneous field with a superimposed square. The stimulus was viewed from a distance of $2 \mathrm{~m}$. The inside of the square $(5.8 \mathrm{~cm})$ subtended a visual angle of $1 \mathrm{deg} 40 \mathrm{~min}$ of arc. The border of the squares $(1.5 \mathrm{~mm})$ subtended a visual angle of $2.6 \mathrm{~min}$ of arc. The latter value has been chosen on the basis of our pilot investigations, which showed vivid illusory distortions for such a border width. Moreover, similar visual angles were used in earlier research (eg Gregory and Heard 1983; Roncato 2000). The square was positioned in the centre of the monitor screen and was viewed through a circular aperture (diameter $15 \mathrm{~cm}$, or $4 \mathrm{deg} 17 \mathrm{~min}$ of arc) positioned in front of the monitor. Either the inside or the outside of the square was subjected to a temporal luminance gradient (the luminance of which ranged from 0.07 to $59.3 \mathrm{~cd} \mathrm{~m}^{-2}$ ) with a frequency of approximately 7 cycles $\min ^{-1}$. These conditions are referred to as VLR-inside and VLR-outside, respectively. The corresponding surfaces without a gradient (CLR-outside and CLR-inside, respectively) and the border of the square could have one of the following 6 luminance values: $0.07,2.11,8.7,20.6,37.6,59.3 \mathrm{~cd} \mathrm{~m}^{-2}$, to be referred to as $\mathrm{L} 1$ (darkest) to L6 (lightest). Note that the lowest and highest possible luminance (L1 and L6) correspond with the lower and upper luminance boundaries of the gradient. With these 6 luminance values, 36 luminance combinations of border and CLR could be formed. As the gradient could be either inside or outside the square this would result in 72 stimuli. In addition, the starting luminance of the gradient was balanced (ie it could either start with L1 or with L6), which produced an actual number of 144 stimuli 
per participant. In figure 3, a trial of the VLR-inside condition and the VLR-outside condition is illustrated.

The task of the subjects was to indicate when they saw the square expanding. The response was to be given by pressing the space-bar of a standard keyboard (a short beep indicated that the response was registered). The subjects were told that they had to press the space-bar when they saw an expanding square. The computer registered during which phase of the temporal gradient the space-bar was pressed, either when the gradient changed from dark to light or when it changed from light to dark. After the space-bar was pressed, the stimulus remained on the screen and the subject was asked to rate the strength of the perceived movement. This rate was to be given on a 5-point scale by pressing one of the corresponding number buttons on the keyboard. The numbers were assigned the following movement strengths: 1, very weak; 2, weak; 3, average; 4, strong; 5, very strong. When no movement was seen at all, an alternative button was pressed (the 'enter' key), after which the next trial appeared on the screen (without a rating being given).

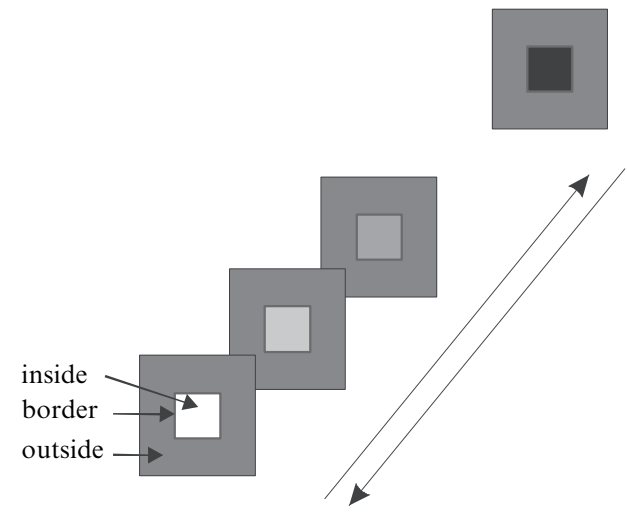

Variable luminance region (VLR): inside

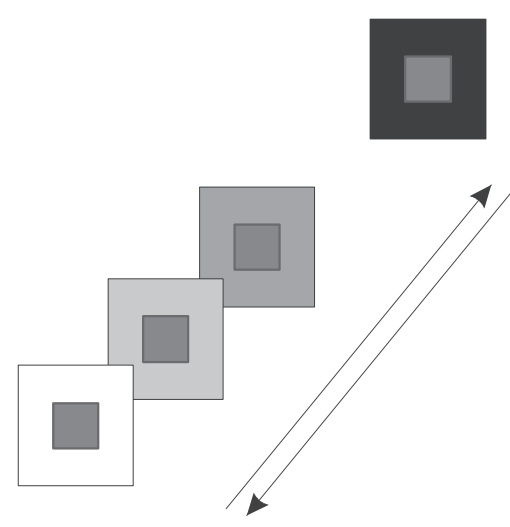

Variable luminance region (VLR): outside

Figure 3. Examples of stimuli of experiment 1a. The VLR-inside condition is shown on the left, and the VLR-outside condition is shown on the right.

During the instruction a few examples were first provided. All subjects spontaneously reported that they saw the squares changing in size. After these initial examples, the procedure and rating were practiced with 25 trials (randomly drawn from the stimulus file).

2.3.2 Experiment $1 b$. In the static displays of experiment $1 \mathrm{~b}$, a spatial gradient was positioned inside or outside a square. The size of the square and of the border, as well as the viewing distance, were the same as in experiment la. Again, the luminance gradient ranged from L1 to L6 (having the same values as in experiment 1a). The position of the luminance gradient was always such that half a period fitted inside the square (see figure $4 \mathrm{a}$, for an example) or outside the square (see figure $4 \mathrm{~b}$, for an example). In the latter case, the luminance of the gradient was continued on the left and the right. The surface with no gradient and the border could have one of 6 luminance values as also used in the dynamic displays of experiment la. The number of stimuli was the same as in experiment la (ie a total of 144), with the phase of the gradient balanced over all trials (ie starting on the left side, the gradient either went from dark to light or from light to dark). This time, the task of the subjects was to indicate which side of the trapezoid-like shapes was perceived to be the longest (left or right), and the response was to be given by pressing one of the arrow keys (leftward pointing versus rightward pointing) of a standard keyboard (again, a short 


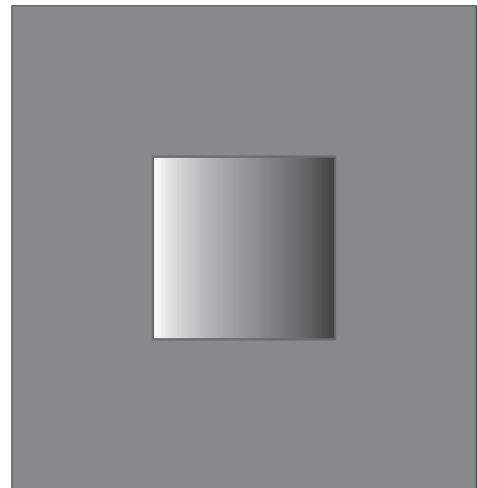

(a) Variable luminance region (VLR): inside

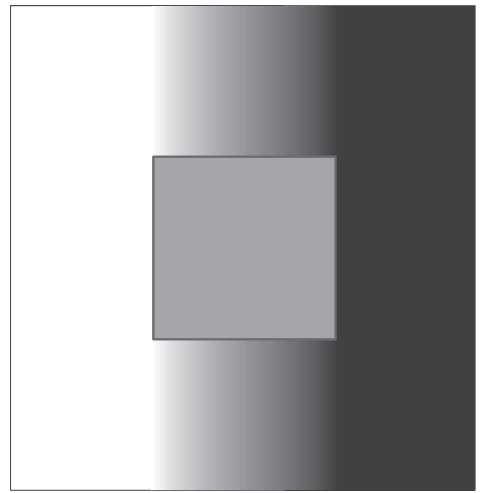

(b) Variable luiminance region (VLR): outside

Figure 4. Examples of stimuli in experiment 1b. The VLR-inside condition is shown on the left, and the VLR-outside condition is shown on the right.

beep indicated that the response was registered). After one of the arrow keys was pressed, the stimulus remained on the screen and the participant was asked to rate the strength of the perceived difference in length. This rate was again to be given on a 5-point scale, similar to experiment la (from 1 to 5; ie from 'very weak' to 'very strong'). In addition, if there was no perceived difference in length, an alternative button was pressed (the 'enter' key), after which the next trial appeared on the screen (without a rating being given).

2.3.3 Experiment 1c. In experiment 1c we focus on the apparent size differences of the bordered squares due to different luminance values of either the inside surface or the outside surface. The actual sizes of the squares and borders, as well as the viewing distances, were the same as in experiments $1 \mathrm{a}$ and 1b. Again, we used the same luminance values as before (experiments $1 \mathrm{a}$ and $1 \mathrm{~b}$ ). This time, each stimulus comprised two bordered squares. In the inside condition, one of the squares had a luminance that was equal to the lower luminance boundary of the gradients used in experiments la and $1 \mathrm{~b}$ (ie luminance value L1) while the other square had a luminance that was equal to the upper luminance boundary of the gradients (ie luminance value L6). The borders of the squares could have one of the 6 luminance values (L1 to L6) and the background could also have one of these 6 luminance values (see figure 5a for an example).

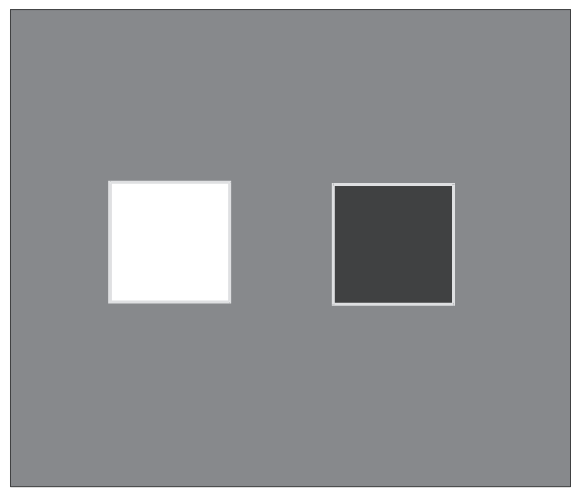

(a) Variable luminance region (VLR): inside

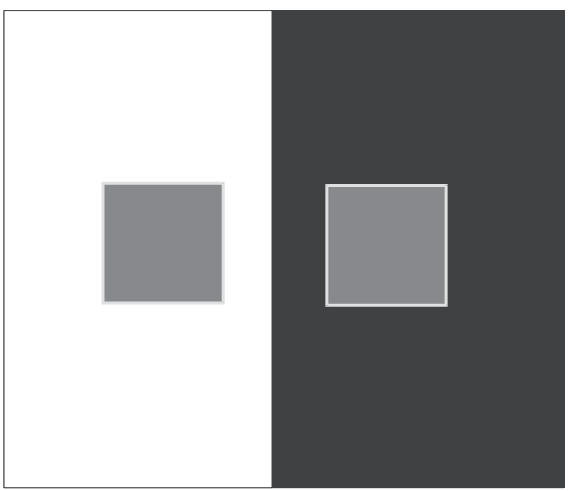

(b) Variable luminance region (VLR): outside

Figure 5. Examples of stimuli of experiment 1c. The VLR-inside condition is shown on the left, and the VLR-outside condition is shown on the right. 
In the outside condition, one of the squares was positioned on a background with the L1 luminance while the other square was positioned on a background with the L6 luminance. Again, the borders could have one of the 6 luminance values and the inside of the squares could also have one of these 6 luminance values (see figure $5 \mathrm{~b}$ for an example). The balancing of conditions was similar to that in experiment $1 \mathrm{~b}$. Note that a few combinations would lead to an entirely dark or entirely light screen (when both the inside and the outside surface and the border would all have luminance value L1 or L6); as a result the total amount of stimuli was $144-8=136$.

This time, the task of the observers was to indicate which square seemed to be larger, the square on the left or the square on the right. As in experiment $1 \mathrm{~b}$, the response was to be given by pressing one of the arrow keys (leftward pointing versus rightward pointing) of a standard keyboard. After that, the rating of the perceived size difference was given on a 5-point scale, as in the previous experiments. If there was no perceived difference in size, the 'enter' key was pressed and no rating was given.

\subsection{Results}

The overall results of experiments $1 \mathrm{a}-1 \mathrm{c}$ are plotted in figure 6. For each panel, on the horizontal axis the border appearance has been plotted. Given the different luminance settings there are three basic conditions plotted on the $x$-axis; the luminance value of the border is lower than the luminance value of the CLR (referred to as the dark-border condition), it can be isoluminant with the CLR (referred to as the no-border condition), or it can be higher than the luminance value of the CLR (referred to as the light-border condition). In addition, the VLR could be inside the square or outside the square (represented by the two lines per panel). The dependent variable at the $y$-axis represents the ratings given by the subjects indicating the strength of the enlargement of the square. For experiment 1a, positive ratings indicate an enlargement of the square when the phase of the VLR gradient changed from dark to light and the negative ratings indicate an enlargement when the phase of the VLR gradient changed from light to dark. For experiment $1 \mathrm{~b}$ positive ratings indicate
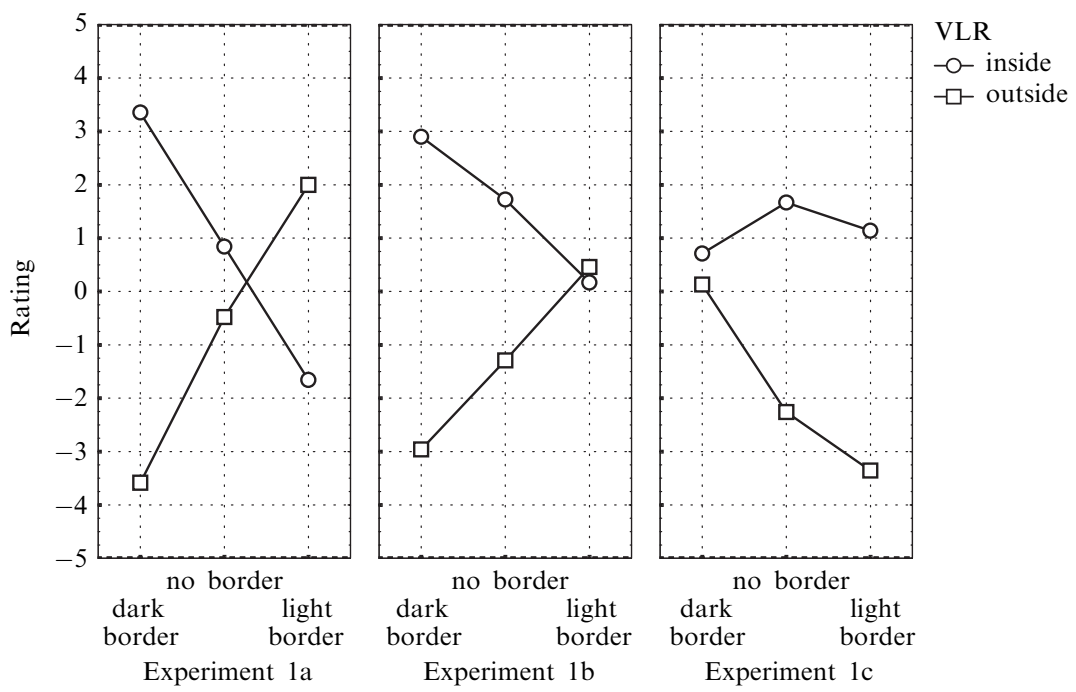

Figure 6. Mean results of experiments 1a, 1b, and 1c. On the horizontal axes the following categories are plotted: dark border - the luminance of the border is lower than the CLR; no-border-the luminance of the border is equal to the CLR; light border - the luminance of the border is higher than the CLR. Separate lines are used for VLR-inside and VLR-outside (VLR: variable luminance region; CLR: constant luminance region). 
an enlargement of that side of the square where the VLR gradient was relatively light and negative ratings indicate an enlargement of that side of the square where the VLR gradient was relatively dark. Finally, for experiment 1c positive ratings indicate relatively larger sizes when the VLR was light, and negative ratings indicate relatively larger sizes when the VLR was dark. Note that in figure 6 the data are pooled per border condition (the luminance of the border could be darker, equal, or lighter than the CLR). The data for each of the specific luminance settings for experiments $1 \mathrm{a}-1 \mathrm{c}$ are shown in the Appendix.

2.4.1 Experiment 1a. For the VLR-inside condition, $t$-tests (on the pooled data, here and further on) reveal that ratings differ from 0 for the no-border, the dark-border, and the light-border condition (no-border: $t_{11}=5.13, p<0.0005$; dark-border: $t_{11}=20.09$, $p<0.0001$; light-border: $\left.t_{11}=10.63, p<0.0001\right)$. The ratings for the dark-border condition and the light-border condition differ significantly from ratings for the no-border condition $\left(t_{11}=18.03, p<0.0001\right.$, and $t_{11}=9.29, p<0.0001$, respectively).

For the VLR-outside condition, the ratings for each border condition differ significantly from 0 (no-border: $t_{11}=4.98, p<0.0005$; dark-border: $t_{11}=27.59, p<0.0001$; light-border: $\left.t_{11}=10.78, p<0.0001\right)$. The ratings for the dark-border condition and the light-border condition differ significantly from the ratings for the no-border condition $\left(t_{11}=9.88, p<0.0001\right.$, and $t_{11}=29.99, p<0.0001$, respectively).

2.4.2 Experiment $1 b$. For the VLR-inside condition, the ratings for the no-border condition differ significantly from $0\left(t_{11}=5.94, p<0.0001\right)$. This holds also for the dark-border condition $\left(t_{11}=12.5, p<0.0001\right)$, but not for the light-border condition $\left(t_{11}=0.30, \mathrm{~ns}\right)$. However, the ratings for the dark-border condition and the light-border condition differ significantly from the ratings for the no-border condition $\left(t_{11}=5.11\right.$, $p<0.001$, and $t_{11}=3.21, p<0.01$, respectively).

For the VLR-outside condition, the ratings for the no-border condition differ significantly from $0\left(t_{11}=4.59, p<0.001\right)$. This holds also for the dark-border condition $\left(t_{11}=10.94, p<0.0001\right)$ but not for the light-border condition $\left(t_{11}=1.58\right.$, ns). Again, the ratings for the light-border condition and the dark-border condition differ significantly from the ratings for the no-border condition $\left(t_{11}=7.16, p<0.0001\right.$, and $t_{11}=5.71, p<0.001$, respectively).

2.4.3 Experiment 1c. For the VLR-inside condition, the ratings for all three conditions (no-border, light-border, dark-border) differ significantly from $0\left(t_{11}=2.71, p<0.05\right.$; $t_{11}=6.86, p<0.0001 ; t_{11}=3.63, p<0.005$, respectively). The ratings for the darkborder condition differ significantly from the ratings for the no-border condition $\left(t_{11}=3.79, p<0.005\right)$, whereas the ratings for the light-border condition do not differ from those for the no-border condition $\left(t_{11}=1.98\right.$, ns).

For the VLR-outside condition, the ratings for the no-border condition differ significantly from $0\left(t_{11}=9.42, p<0.0001\right)$. This holds also for the light-border condition $\left(t_{11}=18.13, p<0.0001\right)$ but not for the dark-border condition $\left(t_{11}=0.63\right.$, ns). The ratings for the light-border condition and the dark-border condition differ significantly from the ratings for the no-border condition $\left(t_{11}=5.79, p<0.0001\right.$, and $t_{11}=7.69, p<0.0001$, respectively).

\subsection{Discussion of experiment 1}

In each of the three plots, there is a significant deviation from 0 for the no-border condition. Generally, in this condition light squares (or lighter parts of the squares) are perceived to be larger than dark squares (or darker parts of the squares) when positioned on the same (greyish) background. Moreover, greyish squares (or parts of the squares) on a dark background are perceived to be larger than when they are positioned on a light background. These general tendencies agree with the irradiation 
effect observed by Helmholtz (1867/1962) who noticed that a light area seems to be larger than a dark area of the same size (see also Weale 1975). Therefore, in judging the effect of the border luminance, the no-border condition should be taken as the baseline.

The pattern of results in experiments $1 \mathrm{a}$ and $1 \mathrm{~b}$ reflects similar tendencies on the influence of the relative border brightness. For the VLR-inside condition, when the borders are relatively dark, there is a perceived enlargement when the gradient changes from dark to light. The rating values decrease when the borders are isoluminant or lighter than the CLR-outside. For the VLR-outside condition this pattern reverses. The common aspect here is that relatively dark borders tend to move (experiment 1a) or bend (experiment 1b) away from the field in which the luminance increases, and to move or bend towards the field in which the luminance decreases. For the relatively light borders the opposite tendencies hold. From the plots in the Appendix for experiments $1 \mathrm{a}$ and $1 \mathrm{~b}$ (see figures A1 and A2) in which the results are shown for all luminance settings, one may further notice that the strengths of the apparent extensions for a given CLR appear to be greater for the lower contrasts between the border and the CLR. Especially when the border is relatively light (positive contrast values), the effects seem to decrease strongly with higher contrast values.

Although the tendencies for the different lines are similar, there are also some noticeable differences between the results of experiments $1 \mathrm{a}$ and $1 \mathrm{~b}$. Overall, the differential effect of the relative brightness of the border as compared to the CLR tends to be larger in experiment la than in experiment $1 \mathrm{~b}$. When the border is darker than the CLR, the ratings are a little higher in experiment la than in experiment 1b. Furthermore, when the border is isoluminant with the CLR, the ratings are much closer to zero in experiment la than in experiment $1 \mathrm{~b}$.

The results of experiments $1 \mathrm{a}$ and $1 \mathrm{~b}$ largely contrast with the results of experiment 1c. In experiment 1c, in the VLR-inside condition, the largest effect arises in the no-border condition. Both darker and lighter borders only seem to weaken the irradiation effect, which can be understood from the fact that the luminance values of these borders are between the luminance values of the light and dark squares. In the VLRoutside condition, the overall influence of relative border brightness is opposite to the effects observed in experiments $1 \mathrm{a}$ and $1 \mathrm{~b}$. There is a perceived enlargement of the square on the dark background when the border and the inside surface are isoluminant. The perceived enlargement is even stronger when the border is lighter than the inside surface and reduces to zero or turns into a small enlargement on the white background when the border is relatively darker than the CLR. Here, light borders further perceptually enlarge grey squares on a dark background (relative to grey squares on a light background), whereas dark borders have the opposite effect. It appears that the perceived enlargement is optimal at large contrasts between the border and the background. As suggested earlier, these findings are in line with Gregory and Heard's (1983) observation that a bar with a light and a dark edge is displaced in the direction of the light edge when the background is darker.

As mentioned before, Roncato (2000) noticed the difference between the static gradient distortions in his study and the luminance-induced perceived displacements in the static displays of Gregory and Heard (1983). Roncato did not, however, mention a possible similarity between his static gradient-induced distortions and Gregory and Heard's dynamic distortions. Gregory and Heard, in turn, did not mention such a possible static analogy of the motion phenomena they observed. Let us focus a little further on this analogy. In figure 7, the directions of the distortions in experiments 1a and $1 \mathrm{~b}$ are illustrated. The figure consists of a VLR, a CLR, and a border between the two regions. The width of the VLR appears relatively larger on the left and relatively smaller on the right (whereas for the CLR the reverse holds). From the present data 
Polarity VLR (-)

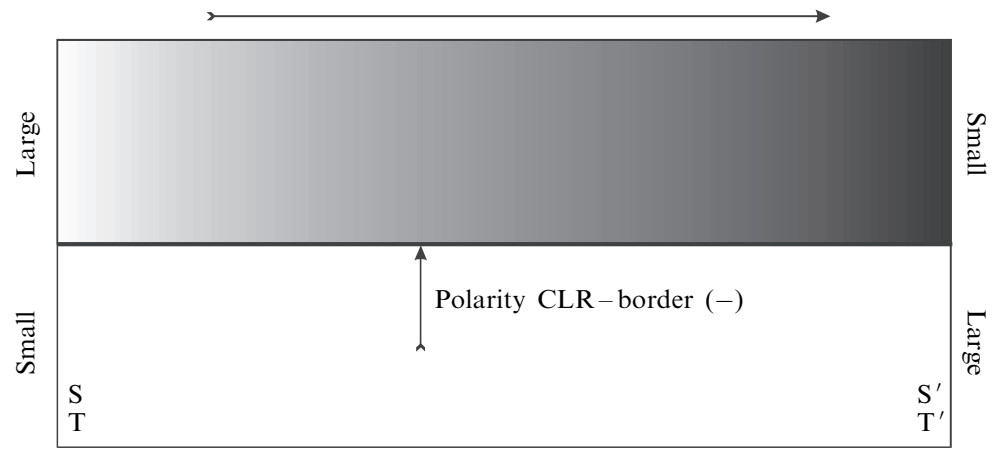

Figure 7. Luminance polarities determine the direction of distortion in a similar way for the dynamic displays of experiment la and the static displays of experiment 1b. Following a VLR surface, it appears smaller in the direction in which the luminance polarity is the same as the luminance polarity from CLR surface to the border. ( $T, \mathrm{~T}^{\prime}$ : points of time; $\mathrm{S}, \mathrm{S}^{\prime}$ : spatial positions; VLR: variable luminance region; CLR: constant luminance region.)

(and from examples such as in, eg, figure 2) it emerges that when the border is lighter than the CLR, the distortion is in the opposite direction. Now, on the basis of the overall data in which the no-border condition is taken as a baseline, the following simple rule for the perceived distortion holds. The VLR appears smaller in the direction where its luminance polarity is the same as when going from the CLR to the border (and the VLR appears larger in the direction where its luminance polarity is opposite to this). In the example of figure 7 the luminance polarity between the CLR and the border is negative, which implies that the VLR would appear smaller in the left - right direction, having a negative polarity as well. Notice that this distortion holds for spatial gradients (with $\mathrm{S}$ and $\mathrm{S}^{\prime}$ referring to spatial positions) and for temporal gradients (with $\mathrm{T}$ and $\mathrm{T}^{\prime}$ referring to points of time).

The above interpretation of our experimental data nicely agrees with the phenomenological observation on the direction of distortion in the Café Wall illusion, and related illusory displays, as has recently been reported by Kitaoka et al (2004). Kitaoka et al noted that such displays can be created by a specific arrangement of elementary units that consists of a solid area and an adjacent line. In particular, Kitaoka et al state that the line appears to bend in a counterclockwise direction when the area and the line have the same polarity (with respect to the background) and in a clockwise direction when the area and the line are of opposite polarity. In our study, such an effect of contrast polarity has been investigated by means of a parametric variation of luminance settings, both spatial and temporal. Moreover, the present data show that this effect should be distinguished from the irradiation effect that might weaken or strengthen the perceived distortion. Obviously, caution should be taken with respect to the polarity rule relating to experiments $1 \mathrm{a}-1 \mathrm{c}$, where the luminance range of the gradient was always from the lowest possible luminance (L1) to the highest possible luminance (L6). To further test its generalisability, other luminance ranges should be tested for both static and temporal distortions. This is done in experiment 2 in which the luminances of the CLR and the border can also lie outside the luminance range of the gradient. In addition, stimuli in which the border of a square has a temporal or a spatial luminance gradient must also be examined. We then also question how the perceived distortions due to a luminance gradient in the border would relate to the perceived distortions due to a luminance gradient in one of the surfaces. 


\section{Experiment 2}

This experiment consisted of two sessions, referred to as experiments $2 \mathrm{a}$ and $2 \mathrm{~b}$, dealing with the same kinds of displays and procedures as in experiments $1 \mathrm{a}$ and $1 \mathrm{~b}$, respectively. As mentioned, there were two additional manipulations in these experiments. We start with the stimulus part that comprises a luminance gradient. As in experiment 1 , the gradient can be attributed to the inside or the outside surface (VLR-inside, VLR-outside), but now the gradient can also be attributed to the border of the square, to be referred to as VLR-border (because of this condition, we will in the following additionally also refer to the 'CLR-border' whenever the border has a constant luminance). Note that a 'replication' of experiment 1c with an additional VLR-border condition is not performed because of the similarities between this condition and the manipulation in experiment 1c, both dealing with surfaces with a constant luminance. Moreover, because of the specific selection of luminance ranges, as explained below, such a 'replication' of experiment 1c with the current luminance settings would lead to a large sample of stimuli with real (ie physically) different sizes of the squares.

As mentioned earlier, the additional manipulation in this experiment concerns the luminance ranges. In experiment 1 , the luminance range of the VLR was always between the lowest luminance value (L1) and the highest luminance value (L6), irrespective of the luminance values of the two rest fields. In this experiment, the lowest and highest luminance values of the gradient are variable too. We selected 4 luminance values; the lowest and the highest value were the same as L1 and L6 of experiment 1, the other 2 values were between L2 and L3, and between L4 and L5 of experiment 1. The luminance values were as follows: $\mathrm{L} 1=0.07 \mathrm{~cd} \mathrm{~m}^{-2}$, $\mathrm{L} 2=5.9 \mathrm{~cd} \mathrm{~m}^{-2}, \mathrm{~L} 3=26.3 \mathrm{~cd} \mathrm{~m}^{-2}$, and $\mathrm{L} 4=59.3 \mathrm{~cd} \mathrm{~m}^{-2}$. Given these values, all pairs of resulting values were used in the experiment. That is, the VLR could be between the values L1 and L2, L2 and L3, or L3 and L4. Additionally, the CLRs could also have the values L1 and L2, L2 and L3, or L3 and L4. This produced 9 different luminance settings ( 3 luminance settings of the CLRs $\times 3$ luminance settings of the VLRs). In fact, this selection of luminance regions comprised three basic conditions involving the relative luminance range between the two CLRs (which could be inside-surface/border, outside-surface/border, or inside-surface/outside-surface) and the VLR (which could be outside-surface, inside-surface, or border, respectively). These relative luminance ranges could be (i) separated (ie CLRs: L1-L2/VLR: L3-L4; CLRs: L3 -L4/VLR: L1 -L2), (ii) joined (ie CLRs: L1 - L2/VLR: L2 - L3; CLRs: L2-L3/VLR: L1-L2; CLRs: L2-L3/VLR: L3-L4; CLRs: L3-L4/VLR: L2 - L3), or (iii) same (ie CLRs: L1 - L2/VLR: L1 - L2; CLRs: L2 - L3/VLR: L2 - L3; CLRs: L3-L4/VLR: L3-L4), see figure 12. Assignment of the luminance values to one of the two CLRs doubled the number of trials to $9 \times 2=18$. Additionally, the phase of the gradient (first-last in experiment $2 a$, or left-right in experiment $2 b$ ) doubles the number to $18 \times 2=36$. Finally, because of the position of the gradient (inside, outside, or border) the total number of trials for each experiment was $36 \times 3=108$. In figure 8 , these conditions are shown schematically.

\subsection{Participants}

Ten subjects participated in experiments $2 \mathrm{a}$ and $2 \mathrm{~b}$. All subjects had normal or corrected-to-normal vision. Half of the subjects first participated in experiment $2 \mathrm{a}$ and then in experiment $2 \mathrm{~b}$. The other half of the subjects went in a reversed order.

\subsection{Apparatus}

The apparatus was the same as in experiment 1. 


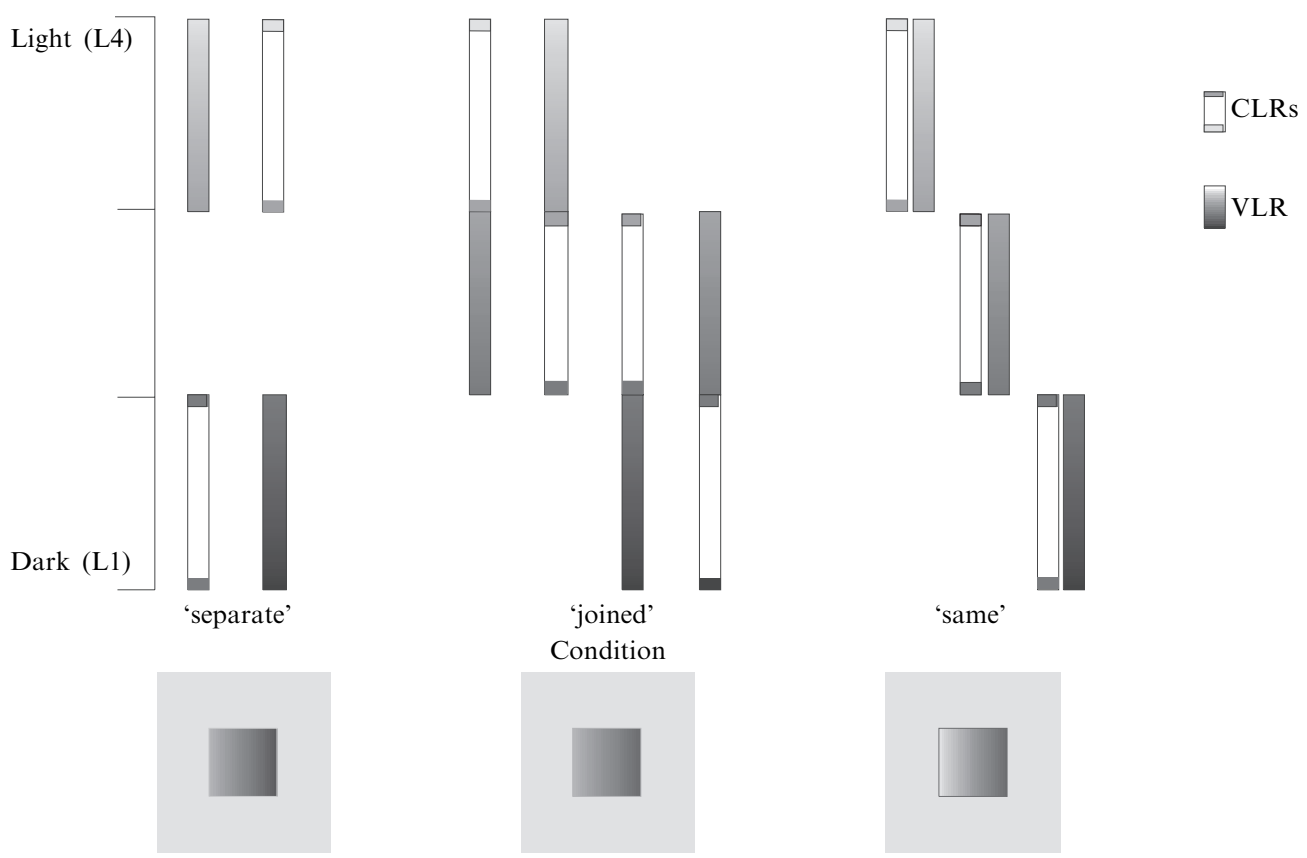

Figure 8. A schematic layout of the relative luminance settings in experiment 2. Each column represents a specific luminance setting. The gradient bars indicate the luminance range of the VLR. The outlined bars (the upper and lower boundary) indicate the luminances of the two CLRs. In the separate condition, the luminance range of the VLR does not include the luminances of the CLRs. In the 'joined' condition, the luminance ranges of the VLR and the CLRs are different but share their highest/lowest luminance values. In the 'same' condition, the luminance ranges of the VLR and the CLRs are the same (VLR: variable luminance region; CLR: constant luminance region).

\subsection{Stimuli and procedure}

3.3.1 Experiment 2a. The subjects viewed a homogeneous field with a superimposed square. The size of the square and of the border, as well as the viewing distance, were the same as in experiment 1. Either the outside surface, the inside square, or the border had a temporal luminance gradient. This gradient cycled between the upper and lower luminance values in about $3 \mathrm{~s}$ per cycle. As in experiment 1a, the task of the subjects was to indicate when they saw the square expanding. Responses were again given by pressing the space-bar on a standard keyboard followed by a rating on a 5-point scale. Again, if no movement was seen, an alternative button was pressed.

3.3.2 Experiment 2b. As in experiment 1b, a spatial gradient was inside or outside a square. Again, the size of the square and the border, as well as the viewing distance, were kept the same as in the previous experiment. In addition, in this experiment, the gradient could be in the border of the square. The position of the surface gradient (inside versus outside) was the same as in experiment $1 \mathrm{~b}$. When the gradient was in the border it was in the horizontal direction.

As in experiment $1 b$, the observers had to indicate which side of the trapezoid-like squares seemed to be the longest (left or right). Responses were again given by the arrow keys, followed by a rating of the strength of the deformation. When there was no perceived difference in length, an alternative button was pressed. 


\subsection{Results}

In figure 9, the results are shown for each of the three VLR conditions for experiment $2 \mathrm{a}$ and experiment $2 \mathrm{~b}$. The dependent variable is again the rating-positive values indicate expansions of the square with increasing luminance, negative values indicate expansion with decreasing luminance. There are two independent variables: luminance difference between the two CLRs (plotted on the horizontal axis) and the relative luminance range $\mathrm{RL}$ (represented by the three lines in each graph: $\mathrm{RL}=1$, separate; $\mathrm{RL}=2$, joined; RL $=3$, same).

3.4.1 Experiment 2a. The VLR-inside condition is shown in the left panel of figure 9a. For the dark-border condition, the positive ratings significantly differ from 0 for all $\mathrm{RL}$ levels (for $\mathrm{RL}=1$ to $3: t_{9}=5.06, p<0.001 ; t_{9}=11.02, p<0.0001 ; t_{9}=14.14$, $p<0.0001$, respectively). For the light-border condition, the positive value for $\mathrm{RL}=1$ differs significantly from $0\left(t_{9}=3.02, p<0.05\right)$ and the negative values for $\mathrm{RL}=2$
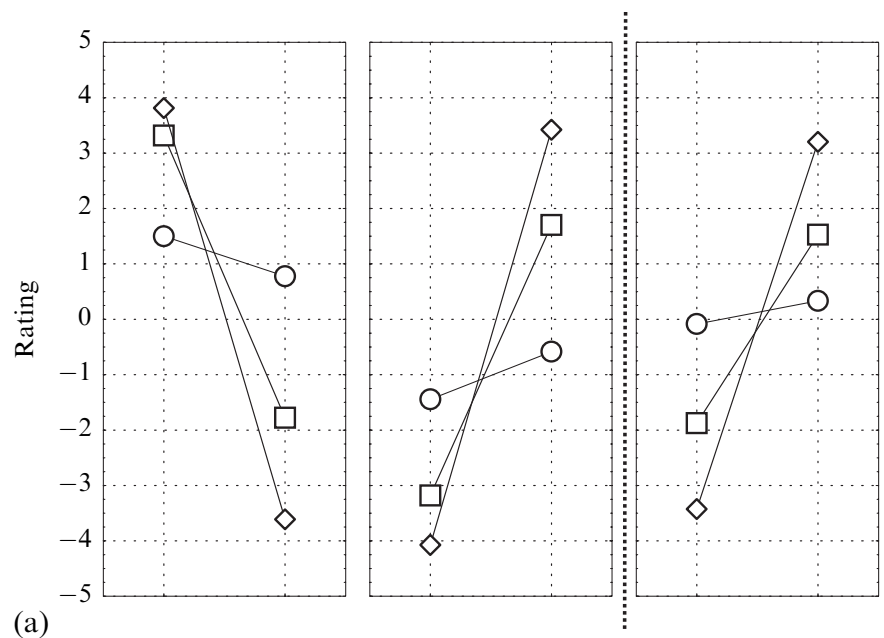

RL

O- 1 (separate)

$\square-2$ (joined)

$\diamond 3$ (same)

(a)
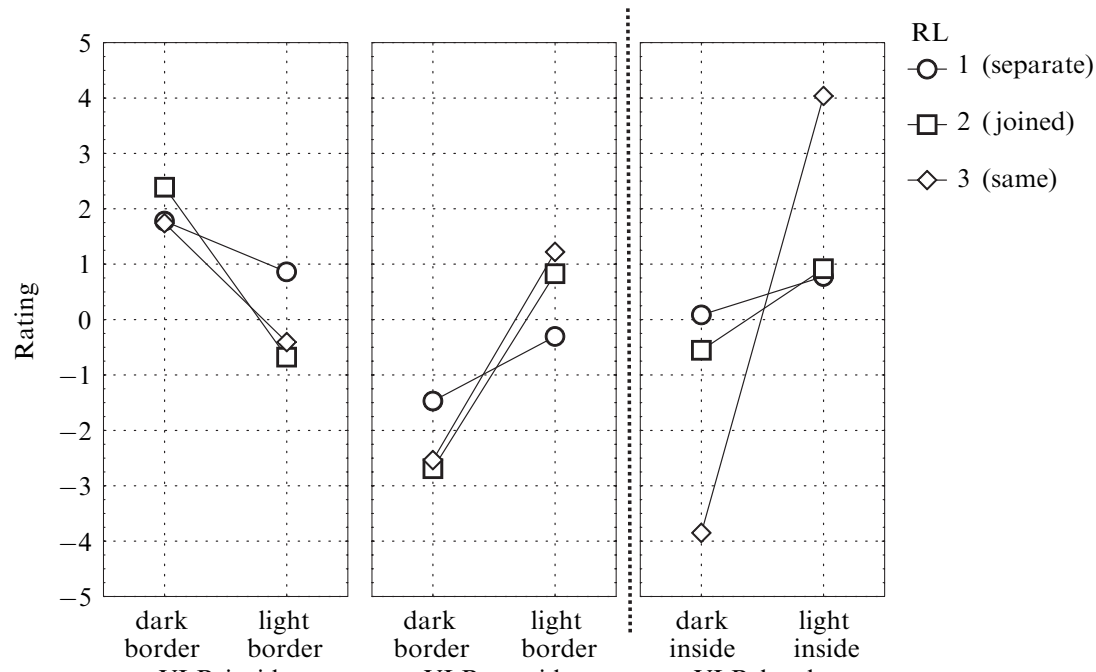

(b)

VLR-inside

VLR-outside

VLR-border

Figure 9. (a) Results of experiment 2a. (b) Results of experiment 2b. Left panel: VLR-inside; middle panel: VLR-outside; right panel: VLR-border. On the horizontal axes the following categories are plotted. Left and middle panel: 'dark borders' versus 'light borders'; right panel: 'dark inside surface' versus 'light inside surface'. The three lines represent the result on the relative luminance ranges $(\mathrm{RL})$ : separate, joined, and same. 
and 3 also differ significantly from $0\left(t_{9}=4.76, p<0.01 ; t_{9}=9.24, p<0.0001\right.$, respectively). In figure $9 \mathrm{a}$ (left panel) it is seen that the lines for the three RL levels are all in the same direction. The differences between dark borders and light borders are significant for all $\mathrm{RL}$ levels (for $\mathrm{RL}=1$ to 3 : $t_{9}=3.12, p<0.05 ; t_{9}=8.09$, $p<0.001 ; t_{9}=12.15, p<0.0001$, respectively).

The VLR-outside condition is shown in the middle panel of figure 9a. For the dark-border condition, the negative ratings significantly differ from 0 for all RL levels (for $\mathrm{RL}=1$ to $3: t_{9}=4.47, p<0.01 ; t_{9}=12.29, p<0.0001 ; t_{9}=16.97, p<0.0001$, respectively). For the light-border condition, the negative value for $\mathrm{RL}=1$ differs significantly from $0\left(t_{9}=2.91, p<0.05\right)$, and the positive values for $\mathrm{RL}=2$ and 3 also differ significantly from $0\left(t_{9}=6.15, p<0.001 ; t_{9}=8.46, p<0.0001\right.$, respectively). The lines for the three RL levels are here also in the same direction. The differences between dark borders and light borders are significant for all RL levels (for RL $=1$ to $3: t_{9}=2.53, p<0.05 ; t_{9}=13.27, p<0.0001 ; t_{9}=10.75, p<0.0001$, respectively).

The VLR-border condition is shown in the right panel of figure 9a. For the dark-inside condition, the rating does not significantly differ from 0 when $\mathrm{RL}=1\left(t_{9}=0.60\right.$, ns), but differs significantly from 0 when $\mathrm{RL}=2$ or $3\left(t_{9}=9.78, p<0.0001 ; t_{9}=11.38\right.$, $p<0.0001$, respectively). In addition, for the light-inside condition, the ratings differ significantly from 0 for all $\mathrm{RL}$ conditions (for $\mathrm{RL}=1$ to $3: t_{9}=4.33, p<0.01 ; t_{9}=6.90$, $p<0.0001 ; t_{9}=10.68, p<0.0001$, respectively). The lines for the three RL levels are all again in the same direction. The differences between dark inside and light inside are significant for all $\mathrm{RL}$ levels (for $\mathrm{RL}=1$ to $3: t_{9}=2.61, p<0.05 ; t_{9}=9.24, p<0.0001$; $t_{9}=11.88, p<0.0001$, respectively).

3.4.2 Experiment $2 b$. The VLR-inside condition is shown in the left panel of figure $9 \mathrm{~b}$. For the dark-border condition, the ratings differ significantly from 0 for all RL levels (for $\mathrm{RL}=1$ to $3: t_{9}=6.62, p<0.0001 ; t_{9}=7.05, p<0.0001 ; t_{9}=4.03, p<0.01$, respectively). For the light-border condition the positive value for $\mathrm{RL}=1$ differs significantly from $0\left(t_{9}=2.98, p<0.05\right)$, the negative value for $\mathrm{RL}=2$ differs significantly from $0\left(t_{9}=2.51, p<0.01\right)$, whereas the rating for $\mathrm{RL}=3$ does not differ significantly from $0\left(t_{9}=0.99\right.$, ns). The lines for the three RL levels are all in the same direction. The differences between dark borders and light borders are significant for all $\mathrm{RL}$ levels (for $\mathrm{RL}=1$ to $3: t_{9}=2.49, p<0.05 ; t_{9}=5.71, p<0.001 ; t_{9}=2.68$, $p<0.05$, respectively).

The VLR-outside condition is shown in the middle panel of figure $9 \mathrm{~b}$. For the dark-border condition, the negative ratings differ significantly from 0 for all RL levels (for $\mathrm{RL}=1$ to $3: t_{9}=5.15, p<0.001 ; t_{9}=10.34, p<0.0001 ; t_{9}=7.14, p<0.0001$, respectively). For the light-border condition the negative value for $\mathrm{RL}=1$ does not differ significantly from $0\left(t_{9}=1.59, \mathrm{~ns}\right)$ whereas the positive values for $\mathrm{RL}=2$ and 3 differ significantly from $0\left(t_{9}=4.55, p<0.01 ; t_{9}=2.40, p<0.05\right.$, respectively). The lines for the three RL levels are all in the same direction. The differences between dark borders and light borders are significant for all RL levels (for $\mathrm{RL}=1$ to 3 : $t_{9}=5.13, p<0.001 ; t_{9}=8.92, p<0.0001 ; t_{9}=5.76, p<0.001$, respectively).

The VLR-border condition is shown in the right panel of figure $9 \mathrm{~b}$. For the dark-inside condition, the rating does not differ significantly from 0 when $\mathrm{RL}=1$ $\left(t_{9}=0.51\right.$, ns) but differs significantly from 0 when $\mathrm{RL}=2$ and $3\left(t_{9}=2.56\right.$, $p<0.05 ; t_{9}=13.43, p<0.0001$, respectively). For the light-inside condition, the ratings differ significantly from 0 for all $\mathrm{RL}$ conditions (for $\mathrm{RL}=1$ to $3: t_{9}=3.58$, $p<0.01 ; t_{9}=2.47, p<0.05 ; t_{9}=17.53, p<0.0001$, respectively). The lines for the three RL levels are all in the same direction. The differences between dark inside and light inside are significant for all $\mathrm{RL}$ levels (for $\mathrm{RL}=1$ to $3: t_{9}=5.84, p<0.001$; $t_{9}=3.30, p<0.01 ; t_{9}=15.85, p<0.0001$, respectively). 


\subsection{Discussion of experiment 2}

Both the VLR-inside condition and the VLR-outside condition of experiments $2 \mathrm{a}$ and $2 \mathrm{~b}$ replicate the basic findings of experiments $1 \mathrm{a}$ and $1 \mathrm{~b}$. Expansions were perceived as extensions for all relative luminance ranges in the same direction, although the differential effects were strongest in the 'same' condition, somewhat weaker in the 'joined' condition, and weakest in the 'separate' condition-but still significant in all cases (for both experiments $2 \mathrm{a}$ and $2 \mathrm{~b}$ ). In the latter condition, where the influence of the border is weakest, the influence of the brightness of the surface, ie the irradiation effect, has the highest impact, as for both dark and light borders the ratings are positive in the VLR-inside conditions and negative in the VLR-outside conditions. That is, irrespective of the relative border luminance, the surface appears to expand when the inside gradient changes from dark to light or when the outside gradient changes from light to dark. Nevertheless, the significant differences between dark and light borders, even for the 'separate' condition, agree with the tendencies found in experiment 1 , and further strengthen the polarity rule (see also figure 7 ) in which the contrast polarities between the CLR-surface and CLR-border on the one hand and the polarity within the VLR-surface on the other hand determine the direction of the distortion in both the dynamic and the static case.

The results for the VLR-border condition in experiments $2 a$ and $2 b$ are also in the same directions for all relative luminance regions. It appears that squares with relatively dark insides and light outsides expand when the border gets darker and that squares with relatively light insides and dark outsides expand whenever the border gets lighter. Again, this holds for all three relative luminance domains (strongest in the 'same' condition, and weakest in the 'separate' condition). These tendencies in the

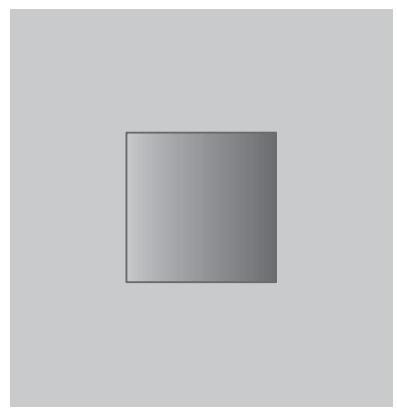

(a)

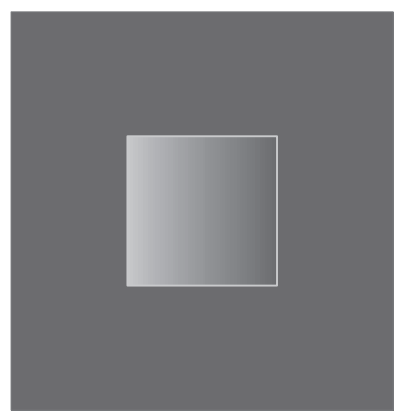

(c)

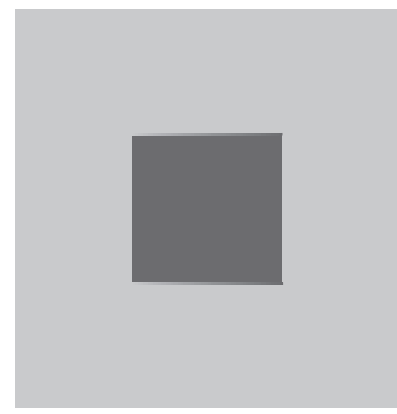

(b)

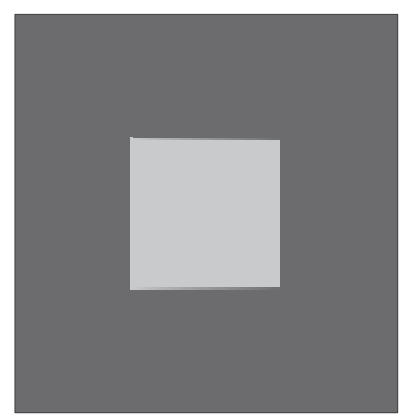

(d)

VLR-inside

Figure 10. Four possible stimuli based on two luminance values and gradients between these values. The displays indicate opposite distortions for panels (a) and (c) versus panels (b) and (d) (see text for further explanation). 
VLR-border condition seem to be opposite to the tendencies in the VLR-surface conditions (like the results of experiment 1c/VLR-outside). We will further illustrate this by a few examples of the 'same' condition of the static displays (see figure 10), as the opposing effects are most pronounced in that case. Displays (a) and (b) of figure 10 each consist of a bordered square on the same light background with the luminance settings of the border and inside surface exchanged: in the VLR-surface condition of display (a), the inside square appears smaller at the side where the luminances approach each other, whereas the opposite applies to the VLR-border condition of display (b). In displays (c) and (d) of figure 10 similar opposed tendencies can be seen for dark backgrounds with, again, an exchange of luminance settings of the border and the inside surface. Note, however, that the present data show that the opposite tendencies apply also to other luminance ranges ('joined' and 'separate'), and are therefore not so much determined by the equality of the actual luminance values at one or the other side but, instead, by polarity differences between, on the one hand, the luminances of the CLRs (CLR-surface versus CLR-border in the VLR-surface condition, and CLR-inside versus CLR-outside in the VLR-border condition) and, on the other hand, the luminance polarity within the VLR. Considering this, it emerges that contrast induction of the border in the VLR-surface condition might account for these opposite tendencies (cf McCourt 1982, 1983). For example, in figures 10a and 10c due to contrast induction the borders appear relatively darker on the left and lighter on the right and, accordingly, in each of these displays, bend towards the lighter region on the left and to the darker region on the right.

\section{General discussion}

The present results show large similarities in distortions of outlined shapes due to luminance gradients in the spatial and temporal domain. Basically, in both domains the distortions are in the same direction. In experiments $1 \mathrm{a}, 1 \mathrm{~b}$, and 2 , it has been shown that in a display where a homogeneous surface, a border, and a surface gradient are present (spatial or temporal), the border inclines in the direction in which the luminance polarity of the gradient is the same as the luminance polarity on transition from the homogeneous luminance surface to the border (figure 7). These shifts can be distinguished from the irradiation effect. The latter is relatively largest in the spatial domain, where the two effects may even neutralise each other in specific cases. In experiment 1c, the irradiation effect appeared to dominate when the size of outlined squares positioned on a homogeneous luminance background had to be compared, quite irrespective of the various luminance settings of border, inside surface, and outside surface. However, when outlined squares with the same inner surface positioned on light and dark backgrounds were compared with each other, the square was perceived to be larger when the contrast between border and background was larger, revealing a direction opposite to the displays with surface gradients. As discussed previously, in experiment 2 distortions due to border gradients were also opposite to distortions due to surface gradients. In addition, we have suggested that contrast induction at the border, in the case of a surface gradient, might explain the opposite border shifts. To get a further grip on the direction of the perceived distortions in the current singlegradient displays, we consider the tendency of non-collinear edges having the same contrast polarity to connect (or join) perceptually, as discussed by Roncato and Casco (2003). We explore two approaches here. In the first approach we determine such polarities based on local differences. In the second approach we determine polarities based on certain global, region-based, differences.

We start with the contrast polarities based on local luminance differences. Consider the display in figure 11a. In that display the gradient is in the border, with the luminance range of the gradient comprising the luminances of the CLRs (as in experiment 1). 
Labelling I
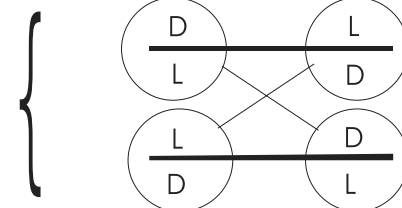

(a)
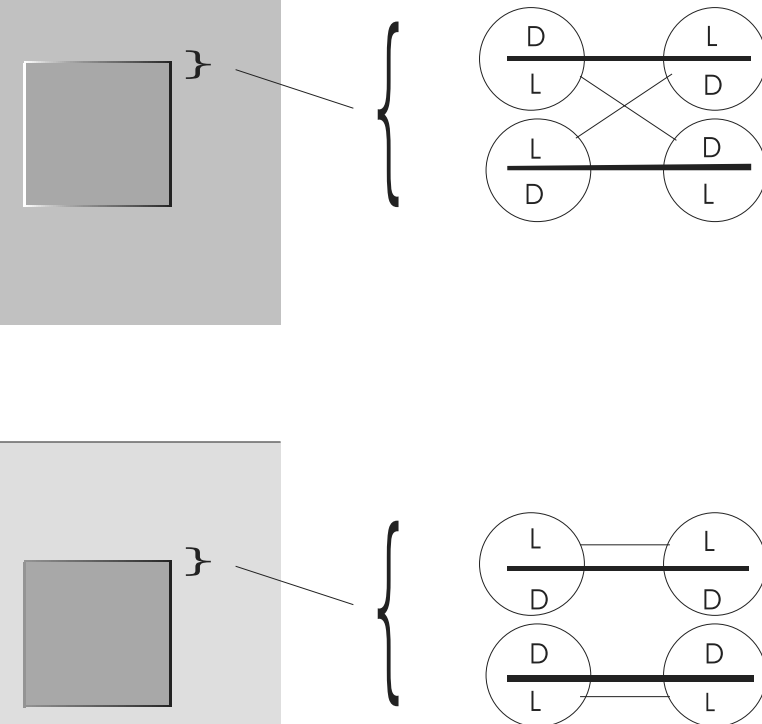

(b)
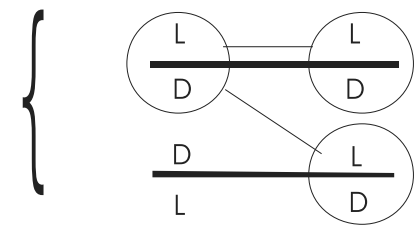

(c)
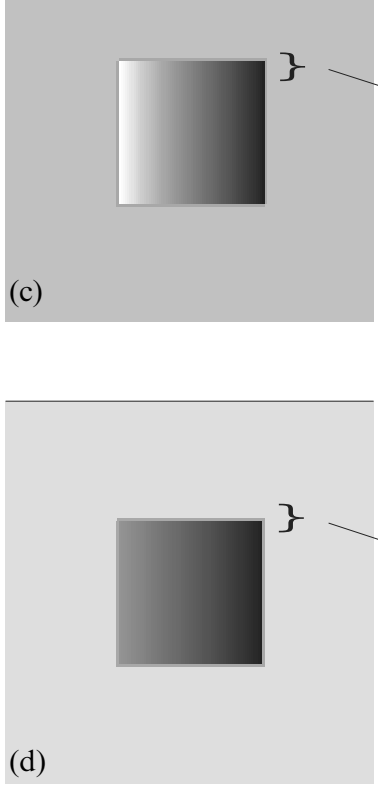
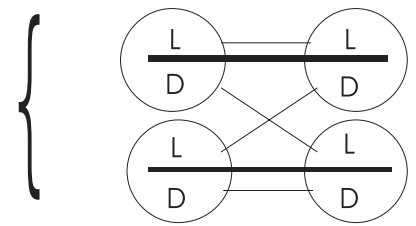

\section{Labelling II}
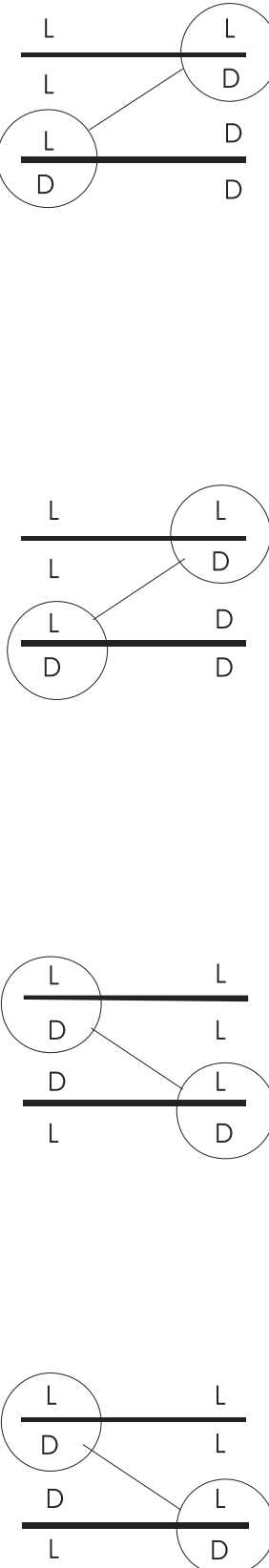

Figure 11. In panels (a) and (c), the luminance range of the gradient (border and surface, respectively) comprises the luminances of the CLRs (comparable to experiment 1). In panels (b) and (d) the luminance range of the gradient does not include the luminances of the CLRs (comparable to the 'separate' condition in experiment 2). Nevertheless, for these panels the direction of the distorting effect seems to be the same when comparing panels (a) and (b) or panels (c) and (d) (see text for further explanation). 
Let us focus here on the upper border, revealing two edges between the surrounding regions; there is one edge between the outer surface and the border and one edge between the border and the inner surface, ie the upper and lower edge. At each edge the local contrast polarity is determined. For example, the leftmost extremity of the upper edge reveals a relatively dark (D) region (the surrounding surface) above a relatively light (L) region (the border itself), whereas the leftmost extremity of the lower edge reveals a relatively light (L) region (the border) above a relatively dark (D) region (the inner surface). These labels are indicated in the second column of figure 11 (Labelling I; in this schematic representation the horizontal lines represent the two edges, with the actual border between the horizontal lines). In a similar way, the contrast polarities are determined for the rightmost extremities. The same has been done for the display in figure 11b, again with a border gradient but now with a luminance range that does not comprise the luminances of the CLRs (as in the separate condition of experiment 2), and also for the displays in figures 11c and 11d with a surface gradient the luminance range of which either comprises the luminances of CLRs (figure 11c) or does not comprise them (figure 11d).

A plausible hypothesis would be that edges with the same contrast polarities tend to be perceived perceptually connected. In fact, Roncato and Casco (2003) suggested and showed such a tendency for specific displays, but also mentioned that other conditions like lateral overlapping of non-collinear edges should be met as well. Here we focus on the predictive account of local contrast polarities. Now, as regards the upper border of the inner square in figure 11a, the top-left and bottom-right contrast polarities are the same and this also holds for the bottom-left and top-right polarities. In figure $11 \mathrm{~b}$, the top-left and top-right contrast polarities are the same, which also holds for the bottom-left and bottom-right polarities. Therefore, on the basis of the local polarities alone we can make no clear predictions of the perceived distortion in figures 1la and 11b. Note that Roncato and Casco would here correctly predict the direction in the display of figure 11a because of an additional analysis on edge overlap. Also in the case of the displays in figures 11c and 11d the labelling reveals different outcomes: in figure 11d all local contrast polarities are the same. Obviously, given the fact that perceived distortions are in the same direction when comparing different luminance ranges of the gradient (including our 'separate' condition, figure 11a versus $11 \mathrm{~b}$, or figure 11c versus 11d), these local edge polarities are not sufficient to predict perceived distortions.

To explore further whether we can deal with the current (single-gradient) displays using the notion of polarity-based edge connections (derived from Roncato and Casco), let us now consider polarities based on certain global brightness differences. The idea is that, given this framework, the $\mathrm{L}$ and $\mathrm{D}$ qualifications should not be determined in relation to each other at a local extremity at opposing sides of an edge but, instead, should be derived from certain global, region-based, comparisons where brightness differences between and within regions (surfaces and borders) are taken into account. Such an alternative labelling could proceed in the following steps: label the VLR (surface or border) extremities according to their relative brightness, label the CLR-surface extremities in relation to the brightness of the other CLR, and, finally, label the CLRborder extremities (if present) after accounting for contrast induction (ie opposed to the direction of the gradient in the VLR-surface). To exemplify this further, let us consider the displays in figure 11 once more. We start with the examples having a border gradient. In figure 11a (third column; Labelling II), the two extremities above the upper edge are both labelled with an L because of the light outside surface (as compared to the dark inside surface). Similarly, both extremities below the lower edge are labelled with a $\mathrm{D}$, because of the dark inner surface (as compared to the light outside surface). 
As the border gradient changes from light on the left to dark on the right, the left extremities in the border (below the upper edge and above the lower edge) are labelled with an $\mathrm{L}$ and the right extremities are labelled with a $\mathrm{D}$ (on the basis of the withinborder comparison). Now, in figure 11a the same labels are found at the bottom-left and top-right extremities, which agrees with the perceived distortion. Obviously, the other border-gradient example, figure 11b, would contain the same labels as figure 11a, which again agrees with the perceived distortion. Now, let us turn to the examples in which there is a gradient in one of the surfaces. In figure 11c, the labelling shows an $\mathrm{L}$ at both extremities above the upper edge (because of the light outside surface as compared to the border). In addition, the extremities below the lower edge are labelled with an $\mathrm{L}$ on the left and a $\mathrm{D}$ on the right because of the surface gradient. With regard to the border, we apply the contrast induction hypothesis as suggested earlier. That is, the lower side of the upper edge and the upper side of the lower edge are labelled with a $\mathrm{D}$ on the left and with an $\mathrm{L}$ on the right. This procedure would reveal the same labels for the top-left and bottom-right extremities, which agrees with the perceived distortion. Obviously, the other surface gradient example, figure 11d, would have the same labels at all extremities as in figure 11c, which again agrees with the perceived distortion.

The above discussion of the notion of polarity-based edge connection primarily shows that a strict local polarity account is not sufficient. Given this framework, for the current single-gradient displays (having various luminance ranges, including the 'separate' condition of experiment 2), a polarity account dealing with certain global brightness differences would lead to better results. Notice that such a procedure does not deal with the strength of the distortion. For example, the irradiation effect appears to be stronger for the present static displays than for the dynamic displays (and irradiation might even overrule the distortions due to the border). Additional factors determining the strength, also deal with specific luminance settings. See the Appendix for the effect of contrast in experiment 1 and for the effect of the relative luminance range (same, joined, separate) in experiment 2 on the perceived strength of the distortion.

There have also been attempts to explain similar illusory effects at the level of physiological processes. For example, with regard to an apparent movement that occurs when a pattern is replaced by its own slightly displaced negative (called 'reversed phi' or 'reversed apparent movement'), Anstis and Rogers (1975) and Rogers and Anstis (1975) considered a spatial summation or neural blurring process resulting from relatively large receptive fields, gathering luminance signals primarily from lowspatial-frequency components. The fact that the illusion in experiments $1 \mathrm{a}$ and $1 \mathrm{~b}$ was strongest at lower contrasts between the fields with constant luminance values (see Appendix) seems to support the role of large receptive fields and indicates that spatial summation might also be attributable to the present phenomenon. To explain perceived tilts in Café Wall like stimuli, Morgan and Moulden (1986) suggested the role of certain bandpass filters. The role of such filters has recently been discussed by Kitaoka et al (2004) who argued that very specific tuning of these filters, both spatially and directionally, is required, leading to still speculative assumptions on the physiological origin of the distortions. Whatever a full physiological explanation might look like, ultimately it should account for the rather systematic tendencies in both the spatial and temporal domain as has been found in this and other related phenomenological studies.

As mentioned already, the present gradient-induced deformations are akin to other well-known illusions in which the position of certain borders seems to be displaced, like the Café Wall illusion (eg Fraser 1908; Gregory and Heard 1979; McCourt 1983; Takeuchi 1997), the Hollow Squares illusion (eg Bressan 1985; Woodhouse and Taylor 1987), or the Münsterberg illusion; see also Roncato (2000). A variety of luminancebased illusions akin to the Café Wall illusion have recently been presented by Kitaoka 
(1998) and by Kitaoka et al (2004). The experimental data presented here agree with their observations on the direction of the distortions. The goal of our study was to compare figural distortions of outlined shapes caused by luminance gradients in both the spatial and the temporal domain. In the displays studied here, distorting effects due to the border could be distinguished from the classical irradiation effect. Most importantly, in the displays considered here, polarity differences determine the direction of distortion in a consistent way for various luminance ranges with spatial and temporal gradients.

\section{Conclusions}

(i) For outlined shapes, the direction of induced distortion is the same for temporal and static surface luminance gradients (eg experiments la and 1b). This confirms the findings of Gregory and Heard (1983) on induced dynamic distortions, and Roncato (2000) on induced static distortions.

(ii) The distortions due to a static or dynamic luminance gradient (eg experiments 1a and $1 \mathrm{~b}$ ) in a surface are opposite to the static distortions caused by homogeneous luminance fields (eg experiment 1c, VLR-outside). This static distortion (also observed by Gregory and Heard 1983) generally has the same direction as those due to border gradients (experiment 2).

(iii) For both static and dynamic distortions due to a surface luminance gradient in outlined shapes the direction of the perceived distortion follows a polarity rule (see also Kitaoka et al 2004, for the role of polarity in a variety of static displays), holding that there is a contraction on the side of the gradient where the luminance polarity is the same as the polarity from the homogeneous luminance region to the border.

(iv) The polarity rule has now been tested on a great variety of luminance settings in both static and dynamic displays and over various luminance ranges, including dissociated luminance ranges (experiment 2, involving cases in which both the minimum and maximum luminance values of the gradient are both lower or higher than the other luminance values in the display), which further strengthens the polarity notion.

(v) When measuring distortions, the distortion due to the border should be dissociated from the distortions due to the classic irradiation effect. In specific cases the distortion due to irradiation appears to induce the strongest deformation and thus could obscure the distortion due to the border (especially so when these distortions are in opposite directions).

(vi) An explanatory framework in which non-collinear edges having the same local edge polarities are assumed to be perceptually connected (as derived from Roncato and Casco 2003) cannot explain distortions at all luminance ranges. With such a framework, taking account of certain global brightness differences would lead to better results for the current single-gradient displays.

Acknowledgments. RvL received a grant from the Royal Netherlands Academy of Arts and Sciences (KNAW). We would like to thank Marco Puts for programming assistance and Charles de Weert for his advice. We also thank an anonymous reviewer and Akiyoshi Kitaoka for their thoughtful remarks. Parts of this research, including the animations of figures 1a to 1c, have been presented at ECVP 2003 and at the Trieste Symposium on Perception and Cognition, 2003.

\section{References}

Anstis S, 1986 "Recovering motion information from luminance" Vision Research 26 147-160

Anstis S, 2001 "Footsteps and inchworms: illusions show that contrast affects apparent speed" Perception $30785-794$

Anstis S, Rogers B, 1975 "Illusory reversal of visual depth and movement during changes of contrast" Vision Research 15957 -961

Anstis S, Rogers B, 1986 "Illusory continuous motion from oscillating positive-negative patterns: implications for motion perception" Perception $15627-640$ 
Anstis S, Smith D, Mather G, 2000 "Luminance processing in apparent motion. Vernier offset and stereoscopic depth" Vision Research $40657-675$

Bressan P, 1985 "Revisitation of the family tie between Münsterberg and Taylor-Woodhouse illusions" Perception $14579-585$

Cavanagh P, Anstis S, 2002 "The boogie-woogie illusion" Perception 31 1005-1011

Fraser J, 1908 "A new visual illusion of visual direction" British Journal of Psychology 2 307-320

Gregory R, Heard P, 1979 "Border locking and the Café Wall illusion" Perception 8 365-380

Gregory R, Heard P, 1983 "Visual dissociations of movement, position, and stereo depth: some phenomenal phenomena" Quarterly Journal of Experimental Psychology A 35 217-237

Helmholtz H von, 1867/1962 Treatise on Physiological Optics volume 3 (New York: Dover, 1962); English translation by J P C Southall for the Optical Society of America (1925) from the 3rd German edition of Handbuch der physiologischen Optik (first published in 1867, Leipzig: Voss)

Kitaoka A, 1998 "Apparent contraction of edge angles" Perception 27 1209-1219

Kitaoka A, Pinna B, Brelstaff G, 2004 "Contrast polarities determine the direction of Café Wall tilts" Perception $3311-20$

McCourt M, 1982 "A spatial frequency dependent grating-induction effect" Vision Research 22 $119-134$

McCourt M, 1983 "Brightness induction and the Café Wall illusion" Perception $12131-142$

Mather G, 1984 "Luminance change generates apparent movement: implications for models of directional specificity in the human visual system" Vision Research 241399 - 1405

Morgan M, Moulden B, 1986 "The Münsterberg figure and twisted cords" Vision Research 26 $1793-1800$

Rogers B, Anstis S, 1975 "Reversed depth from positive and negative stereograms" Perception 4 $193-201$

Roncato S, 2000 "The effect of luminance variation on the apparent position of an edge" Perception \& Psychophysics $\mathbf{6 2} 762-785$

Roncato S, Casco C, 2003 "The influence of contrast and spatial factors in the perceived shape of boundaries" Perception \& Psychophysics $651252-1272$

Takeuchi T, 1997 "The motion analogue of the Café Wall illusion" Perception 26 569-584

Weale R, 1975 "Apparent size and contrast" Vision Research 15949 -955

Woodhouse J, Taylor S, 1987 "Further studies of the Café Wall and Hollow Squares illusions" Perception $16467-471$ 


\section{Appendix}

The plots in this Appendix depict the results for all luminance settings used in experiments $1 \mathrm{a}, 1 \mathrm{~b}$, and $1 \mathrm{c}$. In each plot, mean ratings are shown by means of the luminance values of the constant luminance region (L1-L6, CLR) and the contrast of the border and the CLR. The border-CLR contrast was calculated by the formula $\left(L_{\text {border }}-L_{\mathrm{CLR}}\right) /\left(L_{\text {border }}+L_{\mathrm{CLR}}\right)$ (Michelson contrast). This revealed the following values for stimuli having a relatively light border (and relatively dark CLR) surface: 0.99, 0.98, $0.93,0.89,0.81,0.74,0.62,0.60,0.48,0.40,0.29,0.22$, 0 . Stimuli having a dark border (and light surface) had the appropriate negative-contrast values.

The pattern of the results from these plots reveals the following. First, as in the overall plots of figure 6 , the general tendencies as regards the influence of the borderCLR contrast appear to be highly similar for experiments $1 \mathrm{a}$ and $1 \mathrm{~b}$. That is, for example, for the VLR-inside condition of both experiments, an expansion of the squares with positive border-CLR contrast (ie squares with light border and dark surface) was perceived when the gradient changed from light to dark. This pattern is reversed for the VLR-outside condition. In addition, it is highly noticeable that for both figures A1 and A2, for each luminance value of the CLR, the apparent expansion of the squares seems to be relatively strong at smaller contrasts between the border and the surface, and then decreases gradually with increasing contrast values (positive or negative). In contrast to the foregoing, results for experiment 1c show no clear tendency in the VLR-inside condition, with reversed tendencies for the VLR-outside condition. That is, in the latter case, when the contrast between the border and the surface is positive (ie squares with light border and dark surface), there is a perceived expansion of the squares on dark background. As a further difference with experiments 1a and 1b, a larger contrast between the border and the CLR seems to strengthen the appearance of the illusory expansion.

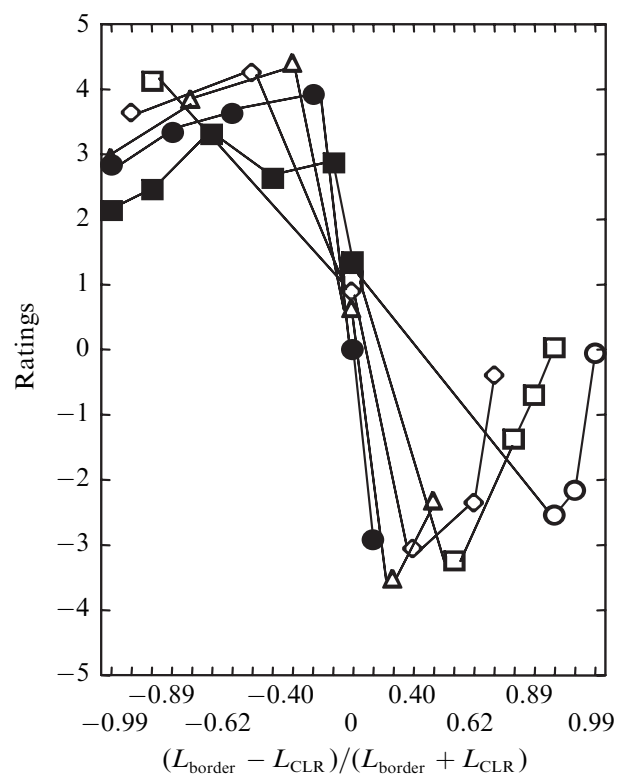

(a) VLR-inside

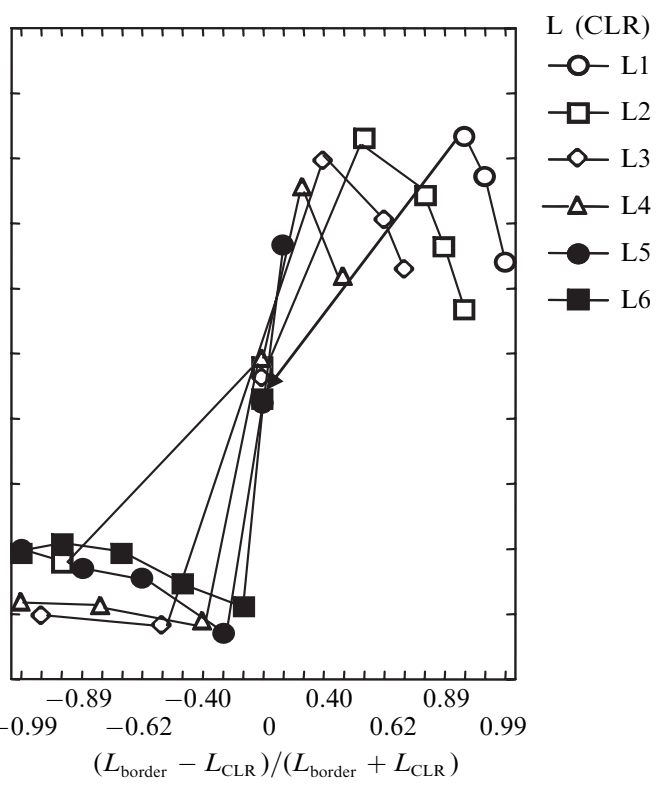

(b) VLR-outside

Figure A1. Results of experiment 1a: (a) the VLR-inside condition; (b) the VLR-outside condition. Positive ratings indicate perceived enlargement of the square when the VLR changes from dark to light. Negative ratings indicate perceived enlargement of the square when the VLR changes from light to dark. Negative contrast values indicate dark border and light surface; positive contrast values indicate light border and dark surface. Separate lines are drawn for each luminance value of the CLR (VLR: variable luminance region; CLR: constant luminance region; L surface luminance). 


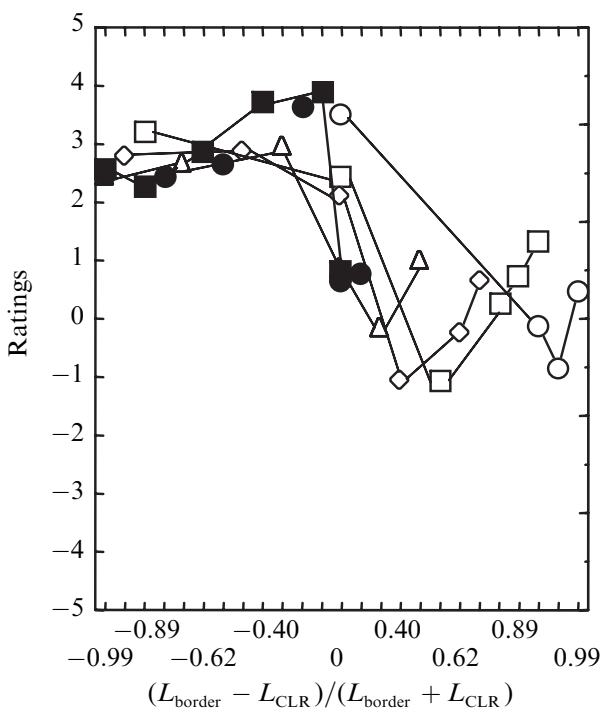

(a) VLR-inside

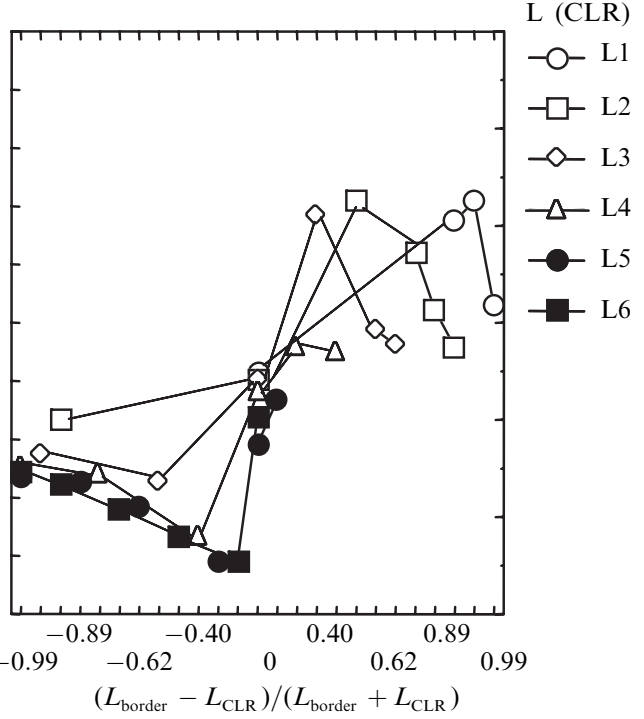

(b) VLR-outside

Figure A2. Results of experiment 1b: (a) the VLR-inside condition; (b) the VLR-outside condition. Positive ratings indicate perceived enlargement of the side of the square where the VLR is relatively light. Negative ratings indicate perceived enlargement of the side of the square where the VLR is relatively dark. Negative contrast values indicate dark border and light surface; positive contrast values indicate light border and dark surface. Separate lines are drawn for each luminance value of the CLR (VLR: variable luminance region; CLR: constant luminance region; L: surface luminance).

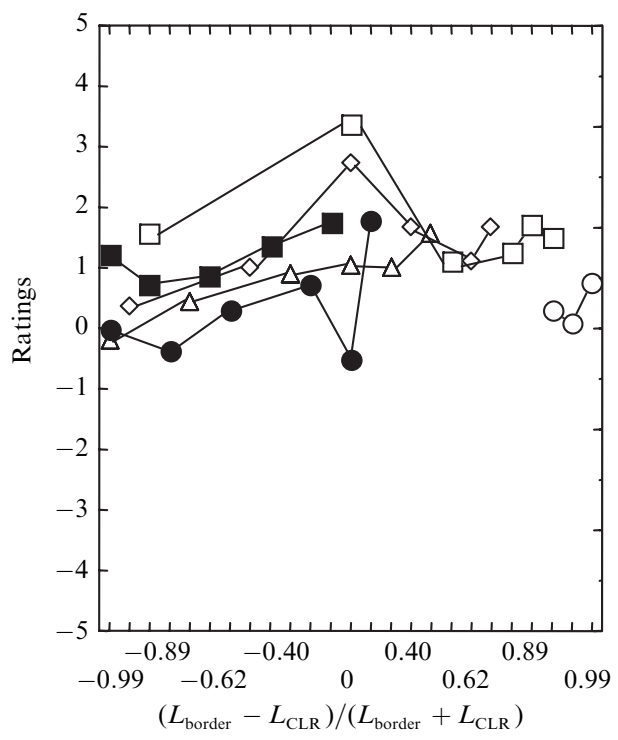

(a) VLR-inside

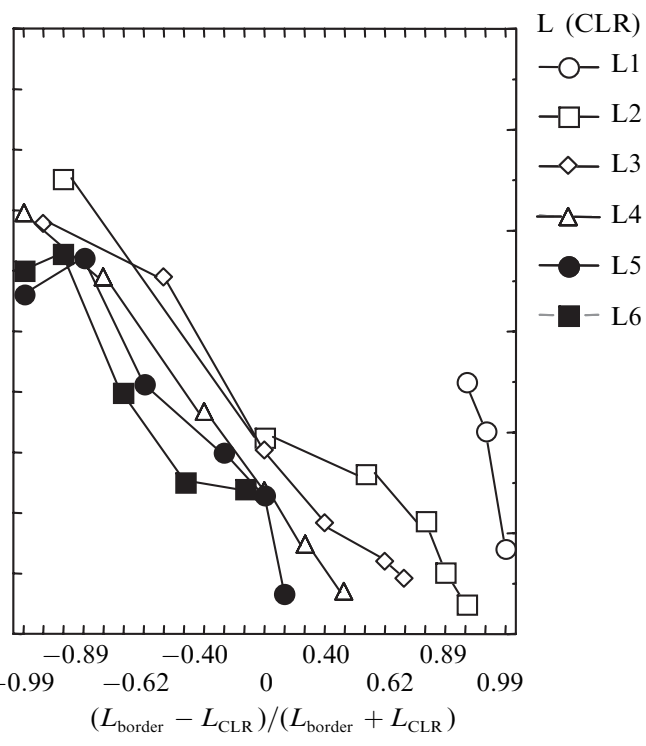

(b) VLR-outside

Figure A3. Results of experiment 1c: (a) the VLR-inside condition; (b) the VLR-outside condition. Positive ratings indicate perceived enlargement of the square when the VLR is relatively light. Negative ratings indicate perceived enlargement of the square when the VLR is relatively dark. Negative contrast values indicate dark border and light surface; positive contrast values indicate light border and dark surface. Separate lines are drawn for each luminance value of the CLR (VLR: variable luminance region; CLR: constant luminance region; L: surface luminance). 


\section{PERCEPTION}

VOLUME 352006

www.perceptionweb.com

Conditions of use. This article may be downloaded from the Perception website for personal research by members of subscribing organisations. Authors are entitled to distribute their own article (in printed form or by e-mail) to up to 50 people. This PDF may not be placed on any website (or other online distribution system) without permission of the publisher. 